\title{
Quantitative electroencephalographic analyses of cardiovascular regulation in low- and high-hostile men
}

\author{
HEATH A. DEMAREE \\ Kessler Medical Rehabilitation Research and Education Corporation, West Orange, New Jersey \\ and \\ DAVID W. HARRISON and ROBERT D. RHODES \\ Virginia Polytechnic Institute and State University, Blacksburg, Virginia
}

\begin{abstract}
This experiment was primarily designed to identify higher cortical correlates of cardiovascular arousal. Low- and high-hostile right-handed undergraduate men were identified using the CookMedley Hostility Scale (CMHS; Cooke \& Medley, 1954). All participants $(N=30)$ completed the coldpressor paradigm. Cardiovascular (heart rate, systolic blood pressure, and diastolic blood pressure) and electroencephalographic (beta magnitude) data were collected before and after the stressor. As predicted, high-hostiles showed greater increases of heart rate and systolic blood pressure to the stressor relative to low-hostiles. The primary findings of this research include significantly greater beta magnitude recorded by the T3, relative to the F7, electrode among low-hostiles. This may suggest that low-hostiles experience left-frontal disinhibition of left-temporal regions, thereby strengthening cardiovascular regulation during the cold-pressor stress. In addition, irrespective of condition, high-hostiles evidenced significantly greater beta magnitude at regions corresponding to the F7 and F8 electrodes. This perhaps suggests that high-hostiles have a relative inability to increase their rostral modulation of posterior systems related to cardiovascular reactivity/regulation.
\end{abstract}

Several lines of research have been pursued in the convergent literatures on the expressive (or behavioral) and physiological dimensions of hostility. In recent reviews, heightened physiological reactivity to a stressor has been a reliable and robust positive correlate of hostility level (e.g., Demaree \& Harrison, 1997b; Hardy \& Smith, 1988; Herridge, Harrison, \& Demaree, 1997; Suarez \& Williams, 1989; Ursin, 1960). While these correlates are well described, much work remains to be completed in the transition from a previously behavioral or cognitive model of hostility into a neuropsychological model of emotion and emotional lability. A basic foundation for the integration and extension of this research may exist within the framework of the study of rage and pseudobulbar palsy (e.g., Demakis, Herridge, \& Harrison, 1994), both examples of negative affective lability. This review outlines previous neuropsychological advances in the understanding of emotion, hostility, and cardiovascular regulation. In addition, much needed research exploring the higher cortical role in these phenomena is presented.

\section{Dominant Right Cerebral Role in Emotion}

Viewing emotional processing from a neuropsychological perspective, the right cerebrum appears dominant

Correspondence should be addressed to D. W. Harrison, Virginia Polytechnic Institute and State University, Department of Psychology, Blacksburg, VA 24061 (e-mail: dwh@vt.edu). in the perception and expression of emotion, and negative emotion in particular. Identification of the dynamic right cerebral mechanisms integral to emotional processing highlights its role in negative emotional expression, including hostility.

Previous research suggests that the right cerebrum is dominant in the perception of visually presented emotional stimuli. For example, Ley and Bryden (1979) tachistoscopically presented five extremely negative and five extremely positive emotional adult faces to "normal," intact right-handed undergraduate men without a history of head trauma. They found a significant left visual field (LVF; right cerebrum) advantage for emotional valence recognition. Subjects were both quicker and more accurate when identifying facial expressions presented to the left relative to the right visual field (RVF; left cerebrum). Similarly, Strauss and Moscovitch (1981) tachistoscopically presented a pair of emotional faces to the left and right visual fields of 40 men and 40 women undergraduates. Subjects were significantly better at determining whether the facial expressions were the same (both positive or negative) or different (one positive, one negative) when presented to the LVF. In addition, subjects were faster and more accurate in their determination of facial valence, positive or negative, in the LVF.

Some research, however, suggests that the right cerebrum is especially important in the mediation of negative affect perception. Mandel, Tanden, and Asthana (1991) studied 10 right-hemisphere-damaged (RHD) in- 
dividuals, 10 left-hemisphere-damaged (LHD) individuals, and 10 normal controls (CTLs). CTLs were significantly more accurate when labeling the emotional expression of each face relative to the LHD group. LHDs, in turn, were significantly better at this task relative to RHDs. Interestingly, a significant group by valence effect was noted. Specifically, relative to RHDs, LHDs had a greater advantage in the identification of negative than positive emotional valences. Hartley, Ireland, Arnold, and Spencer (1991) found that tachistoscopically presented neutral faces were processed faster in the left cerebrum while negative faces were processed more quickly in the right cerebrum. Last, Crews and Harrison (1994) studied 12 depressed and 12 nondepressed right-handed undergraduate men and women. Using a tachistoscope paradigm to present negative, positive, and neutral faces, they found that normal controls showed symmetry in the identification of positive valences. However, a significant right cerebral advantage in the processing of negative emotional faces was found among normals, irrespective of gender. Taken together, this research suggests relative right cerebral dominance for negative emotional perception of visual stimuli.

The motor expression of affect also appears central to right cerebral functioning, further supporting its role in emotional processing. Evidence was first introduced by Campbell (1978), who discovered that smiles were broader on the left side of the face, a region predominantly innervated by the right cerebrum. Borod and Caron (1980) replicated this finding by showing that both positive and negative posed facial expressions were more intense on the left hemiface, with no significant differences found between the subjects' gender ( 26 men, 25 women) or handedness ( 31 right-handed and 20 left-handed). Borod, Koff, Lorch, and Nicholas (1986), in their study of 27 braindamaged individuals ( 12 right-brain-damaged and 15 left-brain-damaged), further found that left cerebrum lesions reduced the use of literal speech, whereas patients with right cerebrum dysfunction evidenced poorer facial affect perception and expression. Indeed, five empirical studies (Blonder, Burns, Bowers, Moore, \& Heilman, 1993; Borod, Koff, Lorch, \& Nicholas, 1985; Borod, Koff, Lorch, Nicholas, \& Welkowitz, 1988; Buck \& Duffy, 1980; Ross \& Mesulam, 1979), using a total of 31 rightbrain-damaged patients, 30 left-brain-damaged patients, and 34 normal controls, independently found that rightbrain-damaged patients were significantly less emotionally expressive in the face than both normal controls and left-brain-damaged patients.

The right cerebrum may, however, be particularly dominant for facial expression of negative affect. Borod, Koff, and Buck (1986) discovered that posed negative facial expressions were rated as more intense on the left hemiface than were positive expressions. Further, Davidson, Ekman, Saron, and Senulis (1990) used quantitative electroencephalography (QEEG) recordings to conclude that spontaneous expressions of disgust and happiness correlated with heightened right- and left- frontotempo- ral activity, respectively. This suggests that the right cerebrum may be dominant in negative affect expression, whereas the left cerebrum may largely control positive affect expression.

Some research suggests that affective gestures are largely controlled by the right cerebrum. Emotional gestures, while largely underacknowledged, are important facets that enrich interpersonal communication. Ross and Mesulam (1979) found that right frontal opercula lesions corresponded with a complete loss of spontaneous gestures. Similarly, Ross (1981) noted that right frontal lesions were associated with decreased use of affective gesture.

\section{Right Cerebrum and Hostility}

Given the above research implicating the right cerebrum as an important processor of emotion, and negative emotion in particular, it is not surprising that the right hemisphere has been linked to hostility expression. Lesion studies have been important contributors to our understanding of hostility. Several anatomical systems have been identified in which dysfunction at specific loci have been correlated with emotional lability, including rage and/or pseudobulbar palsy (uncontrollable extreme outbursts of emotion, including anger and crying). First, bilateral lesions of the bulbar motor nuclei, and the neocortical upper motor neurons in particular, have produced pseudobulbar affect (Lieberman \& Benson, 1977). Second, lesion of the diencephalon, basal forebrain, medial temporal lobe, or the brainstem tegmentum have yielded emotional lability (Brown, 1967; Contu \& Drew, 1966; Sackheim et al., 1982). Ross (1993; Cases 1 and 3) also theorized that pathological affect may result from a combination of major depression and a right frontal opercula lesion, as supported by two case studies.

The hypothalamus was also discovered as an important mediator to rage production (Moyer, 1987; Panksepp, 1982). Ablation of sites in the hypothalamus result in sham rage, suggesting that pathways from the right frontal lobe to the hypothalamus may inhibit emotional lability. Previous experimental lesion studies with cats (Decsi \& Nagy, 1974; Lu, Shaikh, \& Siegal, 1992; Nagy \& Decsi, 1974) and case studies with humans (Flynn, Cummings, \& Tomiyasu, 1988; Sachdev, Smith, Matheson, \& Last, 1992; Tonkonogy \& Geller, 1992) support the hypothalamic role on rage and pseudobulbar palsy. According to this line of research, the right cerebrum appears central to hostility.

One neuropsychological explanation of hypothalamic rage suggests that the anterior right cerebrum may be responsible for the inhibition or regulation of autonomic functioning as well as the expression of hostility. Activation of the frontal lobe, and the orbital-frontal cortex in particular, appears to decrease hostility levels (Butter, Snyder, \& McDonald, 1970). The orbital-frontal cortex has extensive interconnections with the amygdala of the anterior temporal region via the uncinate tract, an inhibitory pathway (see Kandel, Schwartz, \& Jessell, 1991). 
The amygdala has frequently been described as responsible for heightened hostility. Indeed, stimulation of the amygdala has produced a ragelike response (Ursin, 1960), whereas amygdala lesion has produced placid animals (Woods, 1956). Heilman, Bowers, and Valenstein (1993) hypothesized that these two extensively interconnected regions interact with each other, via the uncinate tract, to yield a relatively conservative and stable aggression level among normals. In support of this theory, we have found decreased orbital-frontal and increased right temporal beta activity in homicidal patients using QEEG (Demaree \& Harrison, 1996; Everhart, Demaree, Harrison \& Williamson, in press).

\section{Right Cerebrum and Physiological Regulation/Arousal}

Some previous research suggests that physiological reactivity is mediated by the right cerebrum. Wittling (1990) experimentally showed 25 men and 25 women subjects an emotionally positive film independently to either the RVF and LVF. For all subjects, LVF presentation of the film resulted in significantly higher blood pressure beyond that which followed presentation to the left cerebrum. However, this effect was significantly greater among men than among women. Wittling described these data as evidence of right hemisphere dominance of physiological regulation, but the results may also be explained by the emotional perception literature. That is, the film's emotional context may have been better perceived by the right brain, thereby inducing greater blood pressure reactivity. Herridge et al. (1997), using 50 right-handed male subjects, found that subjects experienced greater galvanic skin response (GSR) on the left hemibody, primarily innervated by the right hemisphere, when making posed emotional faces. Again, this is suggestive of right cerebral control of physiology; however, one must likewise be cautious of inferring too much from this study. Greater left-hemibody GSR reactivity may result from heightened right-hemisphere arousal in the processing of emotional expression. Although very intriguing, both of these studies may confound physiological arousal with emotional perception or expression. From these studies, it may be premature to conclude that the right brain is dominant in physiological regulation.

Research on arousal, independent of emotion, may promote our understanding of physiological asymmetry. One such study, using 50 right-handed male undergraduates, was performed by Demaree and Harrison (1997b). Using a dichotic listening paradigm to assess cerebral asymmetry, these researchers found a significant relationship between right-cerebral arousal and heart rate (HR) reactivity to the cold-pressor test. Yokoyama, Jennings, Ackles, Hood, and Boller (1987) obtained similar results using an attention-demanding task as a stressor, $\mathrm{HR}$ as the indicator of arousal, and dichotic listening procedures for laterality assessment. Other researchers found that individuals with right relative to left temporoparietal lesions had reduced GSR response to startling stimuli independent of emotional content (Heilman,
Schwartz, \& Watson, 1978; Schrandt, Tranel, \& Domasio, 1989). From their data, Heilman et al. (1978) posited that the right cerebrum plays a dominant role in physiological regulation. While these findings do not infer causality, they suggest that the right hemisphere may play a dominant role in physiological arousal. As suggested by the researchers, other deficits from right-cerebral lesion may indicate hypoarousal. For example, several investigators found reductions in reaction time and diminished attentional functioning from right-hemisphere infarct (DeRenzi \& Faglioni, 1965; Heilman \& Van Den Abell, 1979; Howes \& Boller, 1975; Joseph, 1986; Weinstein, 1978).

Given the above research, it is not surprising that the right cerebrum has been found an important contributor to HR elevation. Interestingly, however, decreased HR has corresponded with arousal of the left cerebrum, which has been theorized to suppress right-hemisphere arousal and its subsequent elevating effect on HR (Zamrini et al., 1990). For example, using right-handed men and women, Hugdahl, Franzen, Andersson, and Walldebo (1983) presented visual stimuli to the right hemisphere $(\mathrm{RH})$ to half of their participants and to the left hemisphere (LH) to the other half. They found an anticipatory HR acceleration in the $\mathrm{RH}$ group and an anticipatory deceleration in the $\mathrm{LH}$ group 3-5 sec before stimulus presentation. Similarly, assessing male and female epilepsy patients subsequent to intracarotid barbiturate injection (WADA), Zamrini et al. (1990) found that injection to the left and right intracarotid artery corresponded with increased and decreased HR, respectively. Lane, Novelly, Cornell, Zeitlin, and Schwartz (1988) used WADA testing on subjects with temporal lobe epilepsy. As in Zamrini et al., injection to the left intracarotid artery corresponded with increased HR. However, this effect was significant only among patients with focal left-, but not right-, temporal lobe epilepsy. These researchers concluded that both amytal sodium and a left temporal epileptic focus may have similar suppressing effects on the right hemisphere and HR. Similarly, Oppenheimer, Gelb, Girvin, and Hachinski (1992) found that, during surgery for epilepsy, stimulation of the left- and right-insular region was associated with decreased and increased HR, respectively.

\section{Hostility and Its Correlates}

Continued advances in neuropsychology have refined our knowledge of psychopaths. Krynicki (1978) compared assaultive and nonassaultive male adolescent delinquents using neuropsychological and EEG tests. He found that the former group had a heightened number of abnormal EEG recordings, particularly in the left frontotemporal regions. Other research suggests that male psychopaths show greater motor impulsiveness, poorer verbal memory, attention and concentration difficulties, poorer abstract thinking, and temporal sequencing problems (Berman \& Siegal, 1976), indicative of frontal lobe dysfunction mainly attributable to the right hemisphere. This finding supports the aforementioned right-cerebral theory of hostility (Heilman et al., 1993). 
Because the right cerebrum may be associated with both negative emotion and physiological lability, it is not surprising that hostility has been associated with increased physiological reactivity to stress. Suarez and Williams (1989) found that high-hostile men experienced greater reactivity as measured by $\mathrm{HR}, \mathrm{BP}$, and blood flow during an anagram task accompanied by harassment than did low-hostile men. Greater physiological reactivity to cognitive stressors among high- relative to low-hostiles has robustly been found (Demaree \& Harrison, 1997b: Schneider, Julius, \& Karunas, 1989; Siegman, Anderson, Herbst, \& Boyle, 1992; Smith, Houston, \& Stucky, 1984; Weidner, Friend, Ficarrotto, \& Mendell, 1989). In research with greater external validity, Jamner, Shapiro, Goldstein, and Hug (1991) studied 33 male paramedics during a 24-h period. Both defensiveness and cynical hostility were found to contribute to cardiovascular reactivity, as indicated by HR and BP, during occupational stress. These have been particularly robust findings, as supported by meta-analysis of the hostility-physiological reactivity relationship (Suls \& Wan, 1993).

Hostility also has been associated with increased persistence (decreased rate of return to baseline measures; Kalat, 1992) on physiological measures after stress. Our lab found GSR perseveration after subjects posed angry faces (Herridge et al., 1997). Further, examination of 28 black men led Ernst, Francis, and Enwonwu (1990) to conclude that manifest hostility is negatively associated with recovery of cardiovascular baseline after stress, produced by repeated mental demands.

\section{Variables}

The classifying variable was self-reported hostility level (high or low).

Two classes of dependent variables were used in this experiment. First, physiological measures were SBP, DBP, and HR. Second, beta magnitude $(13-25 \mathrm{~Hz})$ within the right- and left-temporal lobes (from the T4 and T3 regions, respectively) and the right- and left-frontal poles (from the Fp2 and Fp1 regions, respectively) were assessed using EEG.

\section{Hypotheses}

Hypothesis 1: High-hostile subjects will show greater cardiovascular (SBP, DBP, HR) reactivity to stress relative to low-hostile subjects.

Hypothesis 2: Relative to low-hostiles, high-hostile subjects will show a relatively greater increase of righttemporal (T4) beta magnitude to stress.

Hypothesis 3: Relative to low-hostiles, high-hostile subjects will show a relatively reduced increase in right-frontal (F8) beta magnitude to stress.

Hypothesis 4: Relative to low-hostiles, high-hostile subjects will show a relatively reduced increase of left-frontal (F7) beta magnitude to stress.

Hypothesis 5: Relative to low-hostiles, high-hostile subjects will show a relatively reduced increase of lefttemporal (T3) beta magnitude to stress.

\section{Planned Comparisons}

Comparison 1: The two groups will be compared by assessing anterior (Fpl, Fp2, F7, F3, Fz, F4, F8) and posterior (T5, P3, Pz, P4, T6, O1, O2) beta magnitude.

Comparison 2: The two groups will be compared by assessing left- (Fp1, F7, F3, T3, C3, T5, P3, O1) and right(Fp2, F4, F8, C4, T4, P4, T6, O2) cerebral beta magnitude.

\section{METHOD}

\section{Subjects}

Subjects included 143 right-handed men acquired from the undergraduate psychology pool. Subjects self-reported no history of hearing aids, hearing problems (e.g., tubes in the ears or infections), major illness, head injury, or Reynaud's syndrome. Only males were used because it is essential to ensure as much homogeneity as possible within the experiment to draw conclusions based solely on independent variable differences. All subjects received course credit for their involvement in this research. All identifying materials collected from subjects were destroyed after data collection.

Only subjects who did not have a remarkable medical history were eligible for inclusion in the experiment. Subjects with sufficient hemibody preference, based on the Coren, Porac, and Duncan (1979) laterality test, who additionally received the lowest or highest 15 scores on the Cook-Medley Hostility Scale (CMHS; Cook \& Medley, 1954) were assigned to low- and high-hostility groups, respectively.

\section{Self-Report}

During group testing, subjects were first required to read and sign an informed-consent form. A questionnaire assessing medical history was also given. Subjects were then administered the Coren et al. (1979) lateraltiy test to determine hemibody preference. This self-report assessed right $(-1)$ and left $(+1)$ hemibody preference based on reported preferred use of either eye, ear, arm, and leg. Scores on the test range from a possible -13 to +13 , indicating extreme left and right "handedness," respectively. A score of +5 was required for further participation in the experiment.

Subjects were then administered the CMHS. The CMHS is the most often used measure of hostility and shows validity as a predictor of medical, psychological, and interpersonal outcomes (Contrada, 1994).

One hundred forty-three subjects participated in group testing. Among those receiving a score of +5 or greater on the laterality questionnaire and without a remarkable medical history, subjects with the lowest or highest 15 scores on the CMHS comprised the low- and high-hostility groups, respectively.

\section{Apparati}

The laboratory chamber was composed of a sound-attenuated room (Controlled Acoustical Environments) containing a reclining chair. Located in this chamber were the EEG, physiological, and cold-pressor apparati.

Physiological. SBP, DBP, and HR were assessed using the Norelco Healthcare Electronic Digital Blood Pressure/Pulse Meter with Cuff. SBP and DBP was assessed using the Korotkof method. Accuracy of HR is reported to be within $2 \%$ or 1 beat per minute, while $\mathrm{BP}$ is $\pm 3 \mathrm{~mm} \mathrm{Hg}$ of those auscultated.

Cold pressor. The ice water for the CPT was maintained in a small ice cooler (Gott Corp., Model 1916/2) at $4^{\circ} \mathrm{C}$. Water temperature was measured using a standard mercury thermometer (Fisher Scientific, Model 14-985E).

EEG. Apparati necessary for the collection of EEG data included a lycra electrode cap (Electro-Cap International, Inc.), electrode impedance meter (Lexicor Medical Technology, Model 1089 MKII), electrode gel (Electro-Cap International, Inc.), skinprep 
(D. O. Weaver and Co.), and reference electrodes (Electro-Cap International, Inc.). Data were recorded and analyzed using the Lexicor Neurosearch-24 technologies (Lexicor Medical Technology, 1992). The EEG data were converted on line to digital with a Gateway $486 \mathrm{DX}$ computer for display, storage, and analysis. The amplification factor was 32,000 with a sampling rate of 256 samples/second.

\section{Procedure}

High- and low-hostility subjects were invited back for further involvement in the experiment within a 1 -month time period. They entered the laboratory chamber and were requested to read and sign another informed-consent form.

The experiment consisted of three parts-prestress, stress, and poststress phases.

Prestress phase. As the subject leaned forward slightly while raising his arms, a body harness was placed around his chest and fastened in the back using Velcro straps. Then, the distance between the inion (the bony protuberance at the back of the skull) and the nasion (the juncture where the nose meets the forehead) was measured. Each subject's forehead was marked with a grease pencil $10 \%$ of the measured distance above the nasion. The circumference of the subject's head was then measured by passing the measuring tape through the grease mark and inion. If the circumference was greater than $58 \mathrm{~cm}$, a large electrocap was used. Otherwise, a medium electrocap was used. Wearing vinyl gloves, the researcher used an alcohol swab to wipe the subject's earlobes and forehead. After applying a small amount of skinprep to a cotton swab, the researcher lightly abraded the lateral surface of the subject's earlobes. In a firm yet comfortable manner, reference electrodes were placed over the subject's earlobes. Two sponge disks were placed over the two forehead (Fpl and Fp2) electrodes on the electrocap, the disks were placed on either side of the forehead grease mark. The subject was asked to hold the sponge disks in place while the researcher pulled the electrocap over the back of his scalp. The electrocap's straps were attached to the body harness to keep the cap firmly placed. Leads on the electrocap were attached to the reference leads on the earlobes, and the electrocap was plugged into the electroboard. Impedance was $5 \mathrm{k} \Omega$ or below. If not, the researcher reapplied the ear electrodes until the desired impedance was obtained. After a blunt needle was attached to the syringe, the syringe was filled with electrode gel. The syringe was then used to fill all of the electrodes in the electrocap. Each electrode was tested to ensure that it had an impedance of $5 \mathrm{k} \Omega$ or less. If the desired impedance was not obtained for any electrode, techniques were used until the desired impedance had been obtained. EEG data was now ready for collection.

First, however, cardiovascular data were obtained. Subjects were fitted for BP and HR readings. The blood pressure monitor was strapped to each subject's right upper arm. The researcher then gave the following instructions: "Please take about two minutes to become accustomed to your surroundings and relax." After receiving the opportunity to establish a resting baseline, HR, SBP, and DBP data were collected twice in succession at the end of the 2-min period. To determine the accuracy of the readings, a third reading was taken if the first two readings differed by 6 beats per minute (HR) or $10 \mathrm{~mm} \mathrm{Hg}$ for either SBP or DBP.

EEG data were then collected from the subjects. Each subject was first asked to relax and lie motionless in a supine position on a reclining chair within the sound-attenuated chamber. Specifically, the researcher instructed each subject to do the following:

\footnotetext{
Please sit back on this chair, in a relaxed and comfortable manner, with your eyes closed. Sometimes when we are drowsy or relaxed our eyes tend to roll somewhat when they are closed. Because this will affect the way your brainwaves look, try not to let this happen. Try to avoid moving your eyes during this procedure.
}

A high-pass filter was used to eliminate low-frequency artifact caused by respiration and movement. A sampling rate of $256 \mathrm{~Hz}$ was used, $1201-\sec$ epochs were then recorded. The EEG record was collected exactly 1 min after cardiovascular measures had been obtained.

At the end of the 2-min recording period, the subject continued into the stress phase.

Stress phase. Subjects were given the following instructions:

When you are instructed, please place your left hand in the water to a point about one inch above your wrist. You will be asked to keep your hand in the water for $\mathbf{4 5}$ seconds. Although this may be difficult, please try your hardest to keep your hand in the water until instructed to take it out. Do you have any questions? OK, begin.

After $45 \mathrm{sec}$, the subjects were asked to remove their hands from the water.

Poststress phase. HR, SBP, and DBP arousal was then assessed as in the prestress phase. While no preparation was necessary to collect EEG data, all measures were reassessed in the same manner as the prestress phase (i.e., same procedures and verbal instructions).

Subjects were thoroughly debriefed and any questions were answered.

\section{RESULTS}

\section{Descriptive Measures}

To compare low- and high-hostile groups on descriptive measures, $t$ tests were conducted on scores obtained on the CMHS and Coren et al.'s (1979) Laterality Questionnaire. High-hostiles scored significantly higher on the CMHS $(M=33.53, S D=3.03)$ than did low-hostiles $[M=14.53, S D=3.47 ; t(28)=15.04, p<.05]$. Highhostiles $(M=9.80, S D=2.43)$ did not differ from lowhostiles $(M=9.80, S D=2.57 ; t(28)=.00, p>.05]$ on the Coren et al. Laterality Questionnaire.

\section{Cardiovascular Measures}

A multivariate analysis of variance (MANOVA) was performed on the three cardiovascular variables-SBP, DBP, and HR. The results of the MANOVA and independent ANOVAs are depicted in Table 1. All pairwise comparisons were made using Tukey's Studentized range test (Winer, 1971).

A two-factor mixed design ANOVA with the independent factor of group (low- and high-hostile) and with repeated measure on condition (pre- and poststress) was conducted to analyze HR (Table 1). The main effect of group was found to be statistically significant $[F(1,28)=$ $6.26, p<.05]$, with high-hostile subjects having a higher overall HR $(M=68.6, S D=10.12)$ across conditions relative to the low-hostiles $(M=60.63, S D=7.06)$. The main effect of condition was reliable $[F(1,28)=36.62$, $p<.01]$, with subjects having greater HR during the poststress condition. Finally, a group by condition interaction was reliable $[F(1,28)=13.18, p<.01$; see Figure 1]. Within each condition (pre- and poststress), low- and high-hostiles evidenced significantly different heart rates. Each group also experienced a significant increase in HR subsequent to the cold-pressor condition.

For SBP, a two-factor mixed design ANOVA was performed with a fixed factor of group and repeated measure of condition. The main effect of condition was reliable $[F(1,28)=45.49, p<.01]$. That is, across groups, SBP 
Table 1

MANOVA and Independent ANOVA Results for Physiological Measures

\begin{tabular}{|c|c|c|c|c|c|}
\hline Source & $d f$ & SS & $M S$ & $F$ & $p$ \\
\hline $\begin{array}{l}\text { MANOVA } \\
\text { Hypothesis of an o } \\
\text { Hotelling- } \\
\text { Lawley trace }\end{array}$ & all gro & ffect & & & $<.037$ \\
\hline $\begin{array}{l}\text { Independent ANOVA } \\
\text { Heart Rate }\end{array}$ & & & & & \\
\hline $\begin{array}{l}\text { Group } \\
\text { Condition } \\
\text { Group } \times \text { cond. }\end{array}$ & $\begin{array}{l}1,28 \\
1,28 \\
1,28\end{array}$ & $\begin{array}{r}7,616.13 \\
28.03 \\
1,009.20\end{array}$ & $\begin{array}{r}7,616.13 \\
28.03 \\
1,009.20\end{array}$ & $\begin{array}{r}6.26 \\
36.62 \\
13.18\end{array}$ & $\begin{array}{l}<.018 \\
<.001 \\
<.001\end{array}$ \\
\hline $\begin{array}{l}\text { Systolic Blood Pre } \\
\text { Group } \\
\text { Condition } \\
\text { Group } \times \text { cond. }\end{array}$ & $\begin{array}{l}1,28 \\
1,28 \\
1,28\end{array}$ & $\begin{array}{r}1,009.20 \\
4,465.20 \\
598.53\end{array}$ & $\begin{array}{r}1,009.20 \\
4,465.20 \\
598.53\end{array}$ & $\begin{array}{r}1.08 \\
45.49 \\
6.10\end{array}$ & $\begin{array}{l}<.308 \\
<.001 \\
<.019\end{array}$ \\
\hline $\begin{array}{l}\text { Diastolic Blood } \mathrm{Pr} \\
\text { Group } \\
\text { Condition } \\
\text { Group } \times \text { cond. }\end{array}$ & $\begin{array}{l}\text { ure } \\
1,28 \\
1,28 \\
1,28\end{array}$ & $\begin{array}{r}76.80 \\
418.13 \\
.53\end{array}$ & $\begin{array}{r}76.80 \\
418.13 \\
.53 \\
\end{array}$ & $\begin{array}{r}.08 \\
10.95 \\
.01 \\
\end{array}$ & $\begin{array}{l}<.786 \\
<.002 \\
<.906 \\
\end{array}$ \\
\hline
\end{tabular}

Note-MANOVA, multivariate analysis of variance; ANOVA, analysis of variance.

was significantly higher during the poststress condition. In addition, the group by condition interaction was significant $[F(1,28)=6.10, p<.05$; see Figure 2]. The main effect of group did not approach significance (Table 1). During the poststress condition only, high-hostiles evi- denced a significantly higher SBP than low-hostiles. Both groups, however, experienced significant increases of SBP after the cold-pressor stress.

The mixed design ANOVA for DBP with the fixed factor of group and the repeated measure of condition was

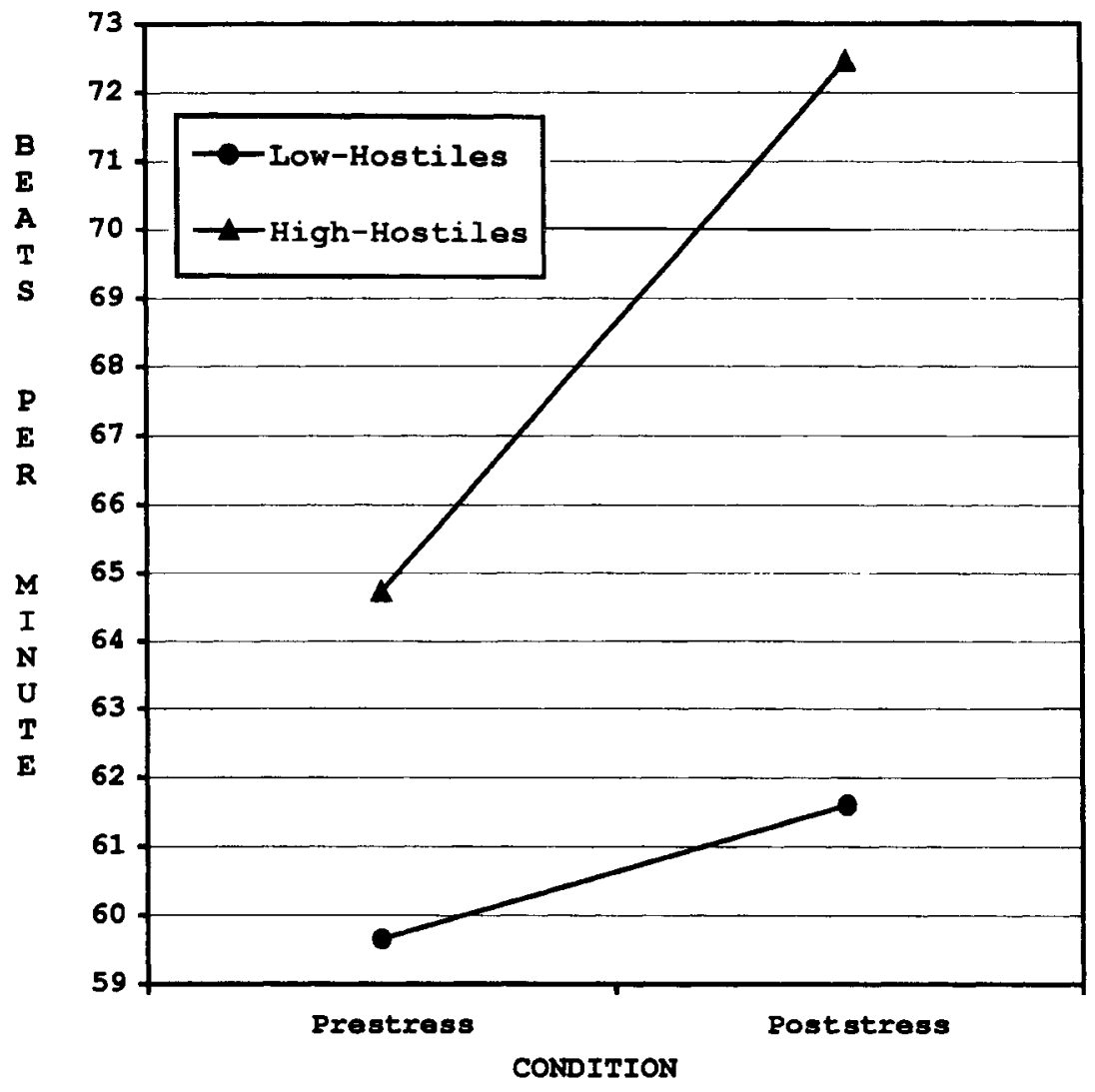

Figure 1. Interaction effect of group by condition on heart rate. 


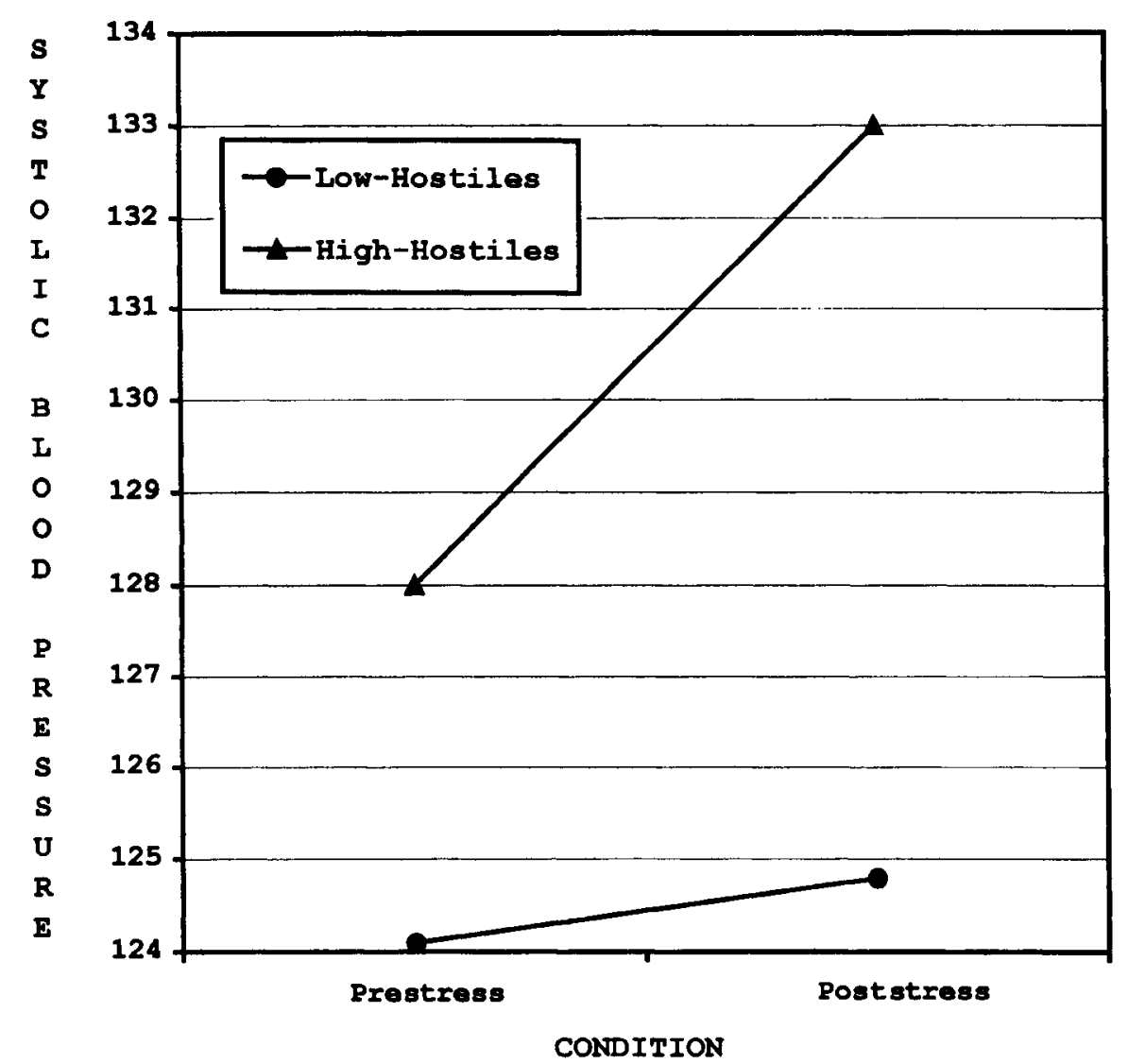

Figure 2. Interaction effect of group by condition on systolic blood pressure.

performed (Table 1). The main effect of condition was significant $[F(1,28)=10.95, p<.01]$, with subjects having greater DBP during the poststress condition.

\section{Quantitative Electroencephalographic Measures}

Hypotheses $2-5$ were assessed by performing a threefactor mixed design ANOVA with a fixed factor of group (low- and high-hostile) and repeated measures of condition (pre- and poststress) and region (F7, F8, T3, and T4). ANOVA results are depicted in Table 2. The interaction of group and region was significant $[F(3,84)=2.79, p<$ .05 ; Table 2, Figure 3]. Across both conditions (both preand poststress), low- and high-hostiles evidenced signif-

Table 2

Summary of the ANOVA Results for Electroencephalographic Measures

\begin{tabular}{lrrrrr}
\hline \multicolumn{1}{c}{ Source } & $d f$ & \multicolumn{1}{c}{ SS } & \multicolumn{1}{c}{$M S$} & \multicolumn{1}{c}{$F$} & \multicolumn{1}{c}{$p$} \\
\hline Group & 1,28 & 33.97 & 33.97 & .30 & $<.585$ \\
Condition & 1,28 & 3.10 & 3.10 & .83 & $<.370$ \\
Group $\times$ cond. & 1,28 & 1.02 & 1.02 & .27 & $<.604$ \\
Region & 3,84 & 19.15 & 6.38 & 1.71 & $<.171$ \\
Group $\times$ region & 3,84 & 31.24 & 10.41 & 2.79 & $<.045$ \\
Region $\times$ cond. & 3,84 & 4.73 & 1.57 & 1.04 & $<.380$ \\
Group $\times$ region & & & & & \\
$\quad \times$ condition & 3,84 & 4.39 & 1.46 & .96 & $<.413$ \\
\hline
\end{tabular}

icantly different beta magnitudes at the $\mathrm{F} 7$ and $\mathrm{F} 8$, but not the T3 and T4, electrode sites.

For a more detailed analysis of this complex interaction, separate and more refined ANOVAs were performed for each group (low- and high-hostiles) using the same regions (F7, F8, T3, and T4) and conditions (pre- and poststress).

For low-hostiles, the main effect of region was statistically significant $[F(3,84)=5.36, p<.01]$. Specifically, the beta magnitude recorded at the F7 electrode was significantly lower than the beta magnitude recorded at the T3 electrode site. No other main or interaction effects were significant.

ANOVAs performed to analyze high-hostile data failed to produce significant main or interaction effects.

\section{Planned Comparisons}

To help assess the role of the anterior, posterior, rightcerebral, and left-cerebral brain regions in cardiovascular regulation (Planned Comparisons 1 and 2), a fivefactor mixed design ANOVA was performed with the fixed factor of group (low- and high-hostile) and with the repeated measures of condition (pre- and poststress), region (left anterior, left posterior, right anterior, and right posterior), cerebrum (left and right cerebrum) and location (anterior and posterior). The main effect of lo- 


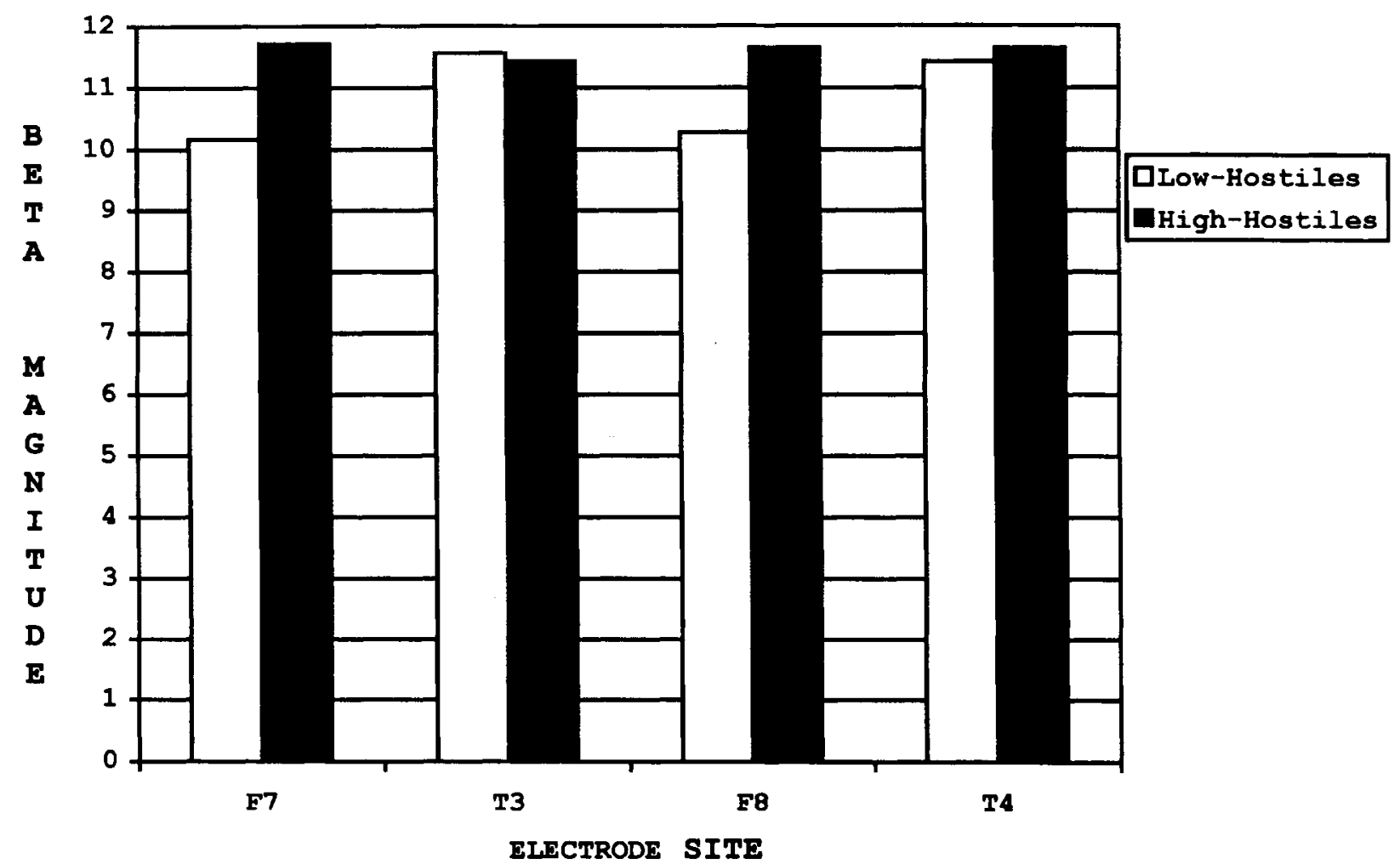

Figure 3. Interaction effect of group by region on electoencephalographic measures.

cation $[F(1,28)=17.16, p<.003]$ was reliable. Specifically, beta magnitudes at posterior regions were significantly greater than those at anterior regions.

\section{DISCUSSION}

In the present research, low- and high-hostile men from an undergraduate population were compared on selfreport, cardiovascular, and QEEG measures. The subjects were assigned to the low- and high-hostile groups by using extreme scores on the CMHS. The robust finding of increased cardiovascular reactivity to the cold-pressor stress was supported by this research. The primary findings include significantly greater beta magnitude recorded by the T3, relative to $F 7$, electrode among low-hostiles. This may suggest that low-hostiles experience left-frontal disinhibition of left-temporal regions, thereby strengthening cardiovascular regulation during the cold-pressor stress. In addition, irrespective of condition, high-hostiles evidenced significantly greater beta magnitude at regions corresponding to the F7 and F8 electrodes. This perhaps suggests that high-hostiles have a relative inability to increase their rostral modulation of posterior systems related to cardiovascular reactivity/regulation.

As predicted, significant main effects of condition were found on the dependent measures of HR, SBP, and DBP. The subjects, irrespective of group, showed greater physiological arousal after the cold-pressor stress. Further, sig- nificant group by condition interaction effects on HR and SBP data were found. These results suggest that highhostiles experienced significantly greater cardiovascular reactivity to the cold-pressor stress on these indicators of cardiovascular arousal (Table 1). This finding of reliable cardiovascular reactivity was restricted to the HR and SBP data. Group differences in DBP reactivity were not reliable.

Hypotheses 2-5 were assessed via a three-factor mixed design ANOVA using the beta magnitude record obtained by the F7, F8, T3, and T4 electrodes. These analyses revealed a significant group by region interaction (Table 2, Figure 3). Low-hostiles, irrespective of condition, evidenced decreased beta magnitude at the regions corresponding to the F7 and F8 electrode sites. This finding of reduced prefrontal beta magnitude among lowhostiles may suggest that they experience a decreased inhibition of temporal beta magnitude (Butter et al., 1970; Demaree \& Harrison, 1997b; Heilman et al., 1993; Ursin, 1960; Woods, 1956). Right- and left-temporal lobe disinhibition has been theorized to produce increased (DeRenzi \& Faglioni, 1965; Heilman \& Van Den Abell, 1979; Howes \& Boller, 1975; Joseph, 1986; Weinstein, 1978) and decreased (Demaree \& Harrison, 1997b; Hugdahl et al., 1983; Lane \& Jennings, 1995; Oppenheimer et al., 1992; Zamrini et al., 1990) cardiovascular arousal, respectively.

Perhaps the most interesting results of this research were found by independently analyzing beta magnitude 
recorded by the F7, F8, T3, and T4 electrode sites by group. An independent ANOVA using high-hostile data only revealed no main (condition or region) or interaction (condition by region) effects. By contrast, an identical analysis of only low-hostile data revealed a significant main effect of region. Specifically, low-hostiles evidenced significantly lower beta magnitude at the $F 7$ relative to the T3 electrode site. This finding may suggest that lowhostiles experience decreased left-frontal beta magnitude, thereby disinhibiting left-temporal beta magnitude (Butter et al., 1970; Demaree \& Harrison, 1997b; Heilman et al., 1993; Ursin, 1960; Woods, 1956). As suggested by a growing literature base (e.g., Demaree \& Harrison, 1997b; Hugdahl et al., 1983; Lane \& Jennings, 1995; Oppenheimer et al., 1992; Zamrini et al., 1990), this lefttemporal disinhibition may produce decreased cardiovascular arousal to stress among low-hostiles. Interestingly, both low- and high-hostiles experienced a nonsignificant difference on inspection and analysis of beta magnitude between regions corresponding to the F8 and T4 electrode sites (Table 2). This may suggest that both lowand high-hostiles experience similar right-brain beta magnitudes, thereby producing like tendencies toward increased cardiovascular arousal to the cold-pressor stress.

At least two possible conclusions may be drawn from low-hostiles' relatively reduced beta magnitude over the F7 and F8 electrode sites (Table 2, Figure 3). First, during the prestress phase, high-hostiles may have approached a "ceiling" on anterior beta magnitude. This ceiling effect may reduce the possibility of further regulation of the posterior systems to the cold-pressor stress among highhostiles. That is, low-hostiles may have increased capacity for anterior modulation of relatively caudal systems. Second, the relative hypoarousal of higher cortical anterior regions among low-hostiles may directly influence subcortical arousal levels, thereby inhibiting cardiovascular reactivity.

The anatomical tracks emanating from the left frontal regions make the rostral inhibition of cardiovascular arousal possible. The left frontal lobe, and the prefrontal cortex in particular, has extensive interconnections with subcortical areas, including the paralamellar portion of the dorsomedial nucleus (DM) of the thalamus and the centromedian-parafascicularis (CM-Pf) complex (Akert \& Von Monakow, 1980; Kievet \& Kuypers, 1977). The paralamellar region of the DM, in turn, has bidirectional bundles with the medulla along the fronto-cortico-dentatorubro-thalamic track. The medulla, through the cranial nerves, controls a number of vital reflexes-breathing, sneezing, coughing, vomiting, and heart rate (Herkenham et al., 1990). Thus, it appears possible that decreased beta magnitude of the left frontal regions may directly influence subcortical structures controlling physiological arousal. In support of this possibility, research robustly suggests that left frontal hypoarousal corresponds with decreased cardiovascular arousal among depressed individuals (Davidson, 1984; Starkstein \& Robinson, 1986).
It should be noted, however, that no significant region by condition interaction effects were found in the singlegroup independent ANOVAs using beta magnitude data recorded by the F7, F8, T3, and T4 electrodes. Given the differential cardiovascular effects of the stressor between groups, this finding is initially difficult to interpret. However, it appears plausible that subjects may have cardiovascularly responded to stress before the administration of the cold-pressor test. Indeed, low- and high-hostiles had significantly different HR and SBP levels during the prestress phase, a finding inconsistent with previous research (Table 1, Figure 2; Demaree \& Harrison, 1997b; Dembroski, MacDougall, Herd, \& Shields, 1979; Glass, McKinney, Hofschire, \& Fedorko, 1990). Previous literature indicates that the preparation of subjects for electroencephalographic recordings may significantly increase their cardiovascular arousal (Knox, 1980). Therefore, the significant group differences in F7, F8, T3, and T4 beta magnitude recordings during both pre- and poststress conditions may reflect disparate higher cortical effects between low- and high-hostiles to the "EEG preparation" stressor. Specifically, relative to high-hostiles, lowhostiles may evidence reduced beta magnitude in the left frontal region before the cold-pressor stress (Table 2, Figure 3 ). This relative hypoarousal may have a disinhibitory effect on left temporal regions (Butter et al., 1970; Demaree \& Harrison, 1997b; Heilman et al., 1993; Ursin, 1960; Woods, 1956), thereby suppressing physiological arousal (Demaree \& Harrison, 1997b; Hugdahl et al., 1983; Lane \& Jennings, 1995; Oppenheimer et al., 1992; Zamrini et al., 1990). Indeed, we suspect that the method used for EEG preparation may produce pain, anxiety, and cardiovascular arousal.

Review of the planned comparisons suggests that, among all subjects, posterior regions had significantly greater beta arousal levels relative to anterior regions. This is not surprising, and is typical among "normal," intact subjects (Talbott, Hales, \& Yudofsky, 1988). This finding may additionally reflect the role of posterior regions, and the reticular activating system in particular, in cardiovascular arousal (Moruzzi \& Magoun, 1949). This region effect may be particularly robust if subjects cardiovascularly responded to the "EEG preparation" stressor experienced before the prestress phase.

This experiment is consistent with prior, related research (Demaree \& Harrison, 1997a, 1997b). Demaree and Harrison (1997b) found that, when administered the cold-pressor stress, low- and high-hostiles experienced reduced and increased right-cerebral arousal, respectively. Cerebral lateralization, however, was assessed using a dichotic listening paradigm. This paradigm most accurately reflects arousal corresponding to the T3 and $\mathrm{T} 4$ regions. The present finding supports the notion that low- and high-hostiles, irrespective of condition, experienced greater beta magnitude at regions assessed by the T3 and T4 electrodes, respectively (Figure 3 ). While these group differences in beta magnitude are unreliable, they 
may produce statistically significant perceptual differences that may be tapped by the dichotic listening paradigm. These differences may additionally produce significant emotional, cognitive, and/or behavioral phenomena that were not assessed during this research. This investigation benefits from the use of technology that specifically reflects beta magnitude of distinct regions, rather than cerebral asymmetry more generally.

This research revealed differential cardiovascular and EEG reactivity to the cold-pressor stress between lowand high-hostiles. One may speculate that low-hostiles' reduced left-frontal beta magnitude may disinhibit lefttemporal arousal and produce a suppressive effect on cardiovascular reactivity to the cold-pressor stress. This research may be important to our understanding of the positive relationship between hostility level and cardiovascular disease (Kubany, Gino, Denny, \& Torigoe, 1994; Lee \& Cameron, 1987; Treiber, Musante, Riley, \& Mabe, 1989). Indeed, knowledge of the higher cortical correlates associated with cardiovascular arousal/regulation may be helpful in devising methods to reduce heightened cardiovascular lability among high-risk populations (e.g., high-hostiles).

While results of this experiment were interesting, limitations in data interpretation persist, and recommendations for improving methodology are also suggested. Generalizations of the present research findings are restricted to men. Future research might incorporate comparisons for sex differences. Additionally, data interpretation is restricted by the lack of convergent dependent measures in the identification of individuals reporting low or high levels of hostility.

In addition to the previously mentioned concerns over data interpretation and generalization, this research could be improved in two important ways. First, because HR and SBP data were collected during pre- and poststress conditions, the group by condition interactions might be attributable to greater cardiovascular perseveration, rather than reactivity, among high-hostiles. This interpretation is consistent with previous hostility research (Ernst et al., 1990; Herridge et al., 1997). Future research may benefit from data collection (cardiovascular and EEG) concurrent with the stress condition. Second, it appears plausible from the results of this research, as well as prior investigation, that subjects may evidence cardiovascular arousal from the experimental preparation for EEG recording. To better evidence higher cortical beta magnitude, changes associated with cardiovascular reactivity, a greater relaxation period between EEG preparation, and prestress measurement of subjects' cardiovascular and beta magnitude baseline appear warranted.

\section{REFERENCES}

AKERT, K., \& VON MONAKOW, K. H. (1980). Relationship of precentral, premotor, and prefrontal cortex to the mediodorsal and intralamminar nuclei of the monkey thalamus. Acta Neurobiologica Experimentica, 40, 7-25.

Berman, A., \& Siegal, A. M. (1976). Adaptive and learning skills in juvenile delinquents: A neuropsychological analysis. Journal of Learning Disabilities, 9, 583-590.

Blonder, L., Burns, A., Bowers, D., Moore, R., \& Heilman, K. M. (1993). Right hemisphere facial expressivity during natural conversation. Brain \& Cognition, 21, 44-56.

Borod, J. C., \& CARON, H. S. (1980). Facedness and emotion related to lateral dominance, sex, and expression type. Neuropsychologia, 18, 237-241.

Borod, J. C., Koff, E., \& Buck, R. (1986). The neuropsychology of facial expression: Data from normal and brain-damaged adults. In P. Blanck, R. Buck, \& R. Rosenthal (Eds.), Nonverbal communication in the clinical context. University Park: Pennsylvania State University Press.

Borod, J. C., Koff, E., Lorch, M. P., \& Nicholas, M. (1985). Channels of emotional communication in patients with unilateral brain damage. Archives of Neurology, 42, 345-348.

Borod, J. C., KofF, E., LorCH, M. P., \& Nicholas, M. (1986). The expression and perception of facial emotion in brain-damaged patients Neuropsychologia, 24, 169-180.

Borod, J. C., Koff, E., Lorch, M. P., Nicholas, M., \& Welkowitz, J. (1988). Emotional and non-emotional facial behaviors in patients with unilateral brain damage. Journal of Neurology, Neurosurgery, \& Psychiatry, 51, 826-832.

Brown, J. W. (1967). Physiology and phylogenesis of emotional expression. Brain Research, 5, 1-14.

Buck, R., \& DufFy, R. J. (1980). Nonverbal communication of affect in brain-damaged subjects. Cortex, 16, 351-362.

ButTer, C. M., SNYder, D. R., \& McDonald, J. A. (1970). Effects of orbital frontal lesions on aversive and aggressive behaviors in rhesus monkeys. Journal of Comparative Physiology \& Psychology, 72, 132-144.

Camprell, R. (1978). Asymmetries in interpreting and expressing a posed facial expression. Cortex, 14, 327-342.

Contrada, R. J. (1994). Personality and anger in cardiovascular disease: Toward a psychological model. In A. W. Siegman \& T. W. Smith (Eds.), Anger, hostility, and the heart. Hillsdale, NJ: Erlbaum.

Contu, R. C., \& Drew, J. M. (1966). Pathological laughing and crying associated with a tumor ventral to the pons. Journal of Neurosurgery, 24, 1024-1026

CooK, W. W., \& MedLey, D. M. (1954). Proposed hostility and pharasaicvirtue scales for the MMPI. Journal of Applied Psychology, 238, 414-418.

Coren, S. P., Porac, C., \& Duncan, P. (1979). A behaviorally validated self-report inventory to assess four types of lateral preferences. Journal of Clinical Neuropsychology, 1, 55-64.

Crews, W. D., \& Harrison, D. W. (1994). Sex differences and cerebral asymmetry in facial affect perception as a function of depressed mood. Psychobiology, 22, 112-116.

Davidson, R. J. (1984). Affect, cognition, and hemispheric specialization. In C. E. Izard, J. Kagan, \& R. B. Zajonc (Eds.), Emotions, cognitions, and behaviors (pp. 320-365). Cambridge: Cambridge University Press.

Davidson, R. J., Ekman, P., Saron, C. D., \& Senulis, J. A. (1990) Approach-withdrawal and cerebral asymmetry: Emotional expression and brain physiology. Journal of Personality \& Social Psychology, 58, 330-341.

DECSI, L., \& NAGY, J. (1974). Chemical stimulation of the amygdala with special regard to the influence on the hypothalamus. Neuropharmacology, 7, 201-207.

Demakis, G. J., Herridge, M. L., \& Harrison, D. W. (1994). Pathological display of positive affect: A bilateral electrodermal case study. Neuropsychiatry, Neuropsychology, \& Behavioral Neurology, 7 , 154-159.

Demaree, H. A., \& Harrison, D. W. (1996). Case study: Topographical brain mapping in hostility following mild closed head injury. International Journal of Neuroscience, 87, 97-101.

Demaree, H. A., \& Harrison, D. W. (1997a). A neuropsychological model relating self-awareness to hostility. Neuropsychology Review, $7,171-185$

Demaree, H. A., \& Harrison, D. W. (1997b). Physiological and neuropsychological correlates of hostility. Neuropsychologia, 35, 1405-1411. 
Dembroski, T. M., MacDougall, J. M., Herd, J. A., \& Shields, J. L. (1979). Effect of level of challenge on pressor and heart rate responses in Type A and B subjects. Journal of Applied Social Psychology, 9, 209-228.

DeRenzi, E., \& Faglioni, P. (1965). The comparative efficiency of intelligence and vigilance tests in detecting hemisphere cerebral damage. Cortex, 1, 410-433.

Ernst, F. A., Francis, R. A., \& Enwonwu, C. O. (1990). Manifest hostility may affect habituation of cardiovascular reactivity in Blacks. Behavioral Medicine, 16, 119-124.

Everhart, D. E., Demaree, H. A., \& Harrison, D. W. \& WilliamSON, J. (in press). "Delusions" of space: A case study utilizing topographical brain mapping and QEEG. Journal of Neuroregulation \& Neurotherapy.

Flynn, F. G., Cummings, J. L., \& Tomiyasu, U. (1988). Altered behavior associated with damage to the ventromedial hypothalamus: A distinctive syndrome. Behavioral Neurology, 1, 49-58.

Glass, D. C., McKinney, M. E., Hofschire, P. J., \& Fedorko, S. (1990). Cardiovascular reactivity to stress: An examination of familial trends. International Journal of Psychophysiology, 9, 1-11.

HARDY, J. D., \& SMITH, T. W. (1988). Cynical hostility and vulnerability to disease: Social support, life stress, and physiological response to conflict. Health Psychology, 7, 447-459.

Hartley, L. R., Ireland, L. K., Arnold, P. K., \& Spencer, J. (1991). Chlorpromazine and the lateralization of the perception of emotion. Physiology \& Behavior, 50, 881-885.

Heilman, K. M., Bowers, D., \& Valenstein, E. (1993). Emotional disorders associated with neurological disease. In K. M. Heilman \& E. Valenstein (Eds.), Clinical neuropsychology (pp. 461-497). New York: Oxford University Press.

Heilman, K. M., Schwartz, H. D., \& Watson, R. T. (1978). Hypoarousal in patients with the neglect syndrome and emotional indifference. Neurology, 28, 229-232.

Heilman, K. M., \& Van Den Abell, T. (1979). Right hemispheric dominance for mediating cerebral activation. Neuropsychologia, 17, 315-322.

Herkenham, M., Lynn, A. B., Little, M. D., Johnson, M. R., Melvin, L. S., deCostA, B. R., \& Rice, K. C. (1990). Cannabinoid receptor localization in brain. Proceedings of the National Academy of Sciences, 87, 164-166.

Herridge, M. L., Harrison, D. W., \& Demaree, H. A. (1997). Differential effects of facial configuration on bilateral skin conductance as a function of hostility. Psychobiology, 25, 71-76.

Howes, D., \& Boller, F. (1975). Simple reaction times: Evidence for focal impairment from lesions of the right hemisphere. Brain, 98 , 317-322.

Hugdahl, K., Franzen, M., Andersson, B., \& Walldebo, G. (1983). Heart-rate responses (HRR) to lateralized visual stimuli. Pavlovian Journal of Biological Science, 18, 186-198.

Jamner, L. D., Shapiro, D., Goldstein, I. B., \& Hug, R. (1991). Ambulatory blood pressure and heart rate in paramedics: Effects of cynical hostility and defensiveness. Psychosomatic Medicine, 53, 393-406.

JoSEPH, R. (1986). Confabulation and delusional denial: Frontal lobe and lateralized influences. Journal of Clinical Psychology, 42, 507-518.

KaLAT, J. W. (1992). Biological psychology. Belmont, CA: Wadsworth.

Kandel, E. R., Schwartz, J. H., \& Jeśsell, T. M. (1991). Principles of neural science. New York: Appleton \& Lange.

KieVET, J., \& KuYPERS, H. G. J. M. (1977). Organization of the thalamocortical connections to the frontal lobe in the rhesus monkey. Experimental Brain Research, 29, 299-332.

KNOX, S. S. (1980). Effect of passive concentration as instruction set for training enhancement of EEG alpha. Perceptual \& Motor Skills, 51, $767-775$.

KRYNICKI, W. E. (1978). Cerebral dysfunction in repetitively assaultive adolescents. Journal of Nervous \& Mental Disease, 166, 59-67.

Kubany, E. S., Gino, A., Denny, N. R., \& Torigoe, R. Y. (1994). Relationship between cynical hostility and PTSD among Vietnam veterans. Journal of Traumatic Stress, 7, 21-31

LANE, R. D., \& JeNNINGS, J. R. (1995). Hemispheric asymmetry, autonomic asymmetry, and the problem of sudden cardiac death. In R. J. Davidson \& K. Hugdahl (Eds.), Brain asymmetry (pp. 271-304). Cambridge, MA: MIT Press.
Lane, R. D., Novelly, R., Cornell, C., Zeitlin, S., \& Schwartz, G. (1988). Asymmetrical hemispheric control of heart rate. Paper presented at the annual meeting of the Society for Psychophysiological Research, San Francisco, and the annual meeting of the American Psychiatric Association, San Francisco.

LeE, M. A., \& Cameron, O. G. (1987). Anxiety, Type A behavior, and cardiovascular disease. International Journal of Psychiatry in Medicine, 16, 123-129.

LEY, R. G., \& BRYDEN, M. P. (1979). Hemispheric differences in processing emotions and faces. Brain \& Language, 7, 127-138.

Ljeberman, A., \& Benson, D. F. (1977). Control of emotional expression in pseudobulbar palsy. Archives of Neurology, 34, 717-719.

Lu, C., Shaikh, M. B., \& Siegal, A. (1992). Role of NMDA receptors in hypothalamic facilitation of feline defensive rage elicited from the midbrain periaqueductal gray. Brain Research, 581, 123-132.

Mandel, M. K., Tanden, S. C., \& Asthana, H. S. (1991). Right brain damage impairs recognition of negative emotions. Cortex, 27, 247-253.

MorUZZI, G., \& MAGOUN, H. W. (1949). Brainstem reticular formation and the activation of the EEG. Electroencephalography \& Clinical Neurophysiology, 1, 455-473.

MOYER, K. E. (1987). Violence and aggression: A physiological perspective. New York: Paragon.

NAGY, J., \& DECSI, L. (1974). Location of the site of the tranquilizing action of diazepam by intralimbic application. Neuropharmacology, 12, 757-768.

Oppenheimer, S. M., Gelb, A., Girvin, J. P., \& Hachinski, V. C. (1992). Cardiovascular effects of human insular cortex stimulation. Neurology, 42, 1727-1732.

PANKSEPP, J. (1982). Toward a general psychobiological theory of emotions. Behavioral Brain Sciences, 5, 407-467.

Ross, E. D. (1981). The aprosodias: Functional-anatomic organization of the affective components of language in the right hemisphere. $\mathrm{Ar}$ chives of Neurology, 38, 561-589.

Ross, E. D. (1993). Nonverbal aspects of language. Neurology Clinics: Behavioral Neurology, 11, 9-23.

Ross, E. D., \& MESUlaM, M. M. (1979). Dominant language functions of the right hemisphere? Prosody and emotional gesturing. Archives of Neurology, 36, 144-148.

Sachdev, P., Smith, J. S., Matheson, J., \& Last, P. (1992). Amygdalohippocampectomy for pathological aggression. Australian \& New Zealand Journal of Psychiatry, 26, 671-676.

Sackheim, H. A., Greenberg, M. S., Weiman, A. L., Gur, R. C., Hungerbuhler, J. P., \& Geschwind, N. (1982). Hemispheric asymmetry in the expression of positive and negative emotions. Archives of Neurology, 39, 210-218.

Schneider, R. H., Julius, S., \& Karunas, R. (1989). Ambulatory blood pressure monitoring and laboratory reactivity in Type A behavior and components. Psychosomatic Medicine, 51, 290-305.

Schrandt, N. J., Tranel, D., \& Domasio, H. (1989). The effects of total cerebral lesions on skin conductance response to signal stimuli Neurology, 39, 223.

Siegman, A. W., Anderson, R., Herbst, J., \& Boyle, S. (1992). Dimensions of anger-hostility and cardiovascular reactivity in provoked and angered men. Journal of Behavioral Medicine, 15, 257-272.

Smith, T. W., Houston, B. K., \& Stucky, R. J. (1984). Type A behavior, irritability, and cardiovascular response. Motivation \& Emotion, $8,221-230$

Starkstein, S. E., \& Robinson, R. G. (1986). Cerebral lateralization in depression. American Journal of Psychiatry, 143, 1631.

Strauss, E., \& Moscovitch, M. (1981), Perception of facial expressions Brain \& Language, 13, 308-332.

Suarez, E. C., \& Williams, R. B. (1989). Situational determinants of cardiovascular and emotional reactivity in high and low hostile men. Psychosomatic Medicine, 51, 404-418.

SULS, J., \& WAN, C. K. (1993). The relationship between trait hostility and cardiovascular reactivity: A quantitative review and analysis. Psychophysiology, 30, 615-626.

Talbott, J. A., Hales, R. E., \& Yudofsky, S. C. (1988). Textbook of psychiatry. Washington, DC: American Psychiatric Press.

TonKonOGY, J. M., \& Geller, J. L. (1992). Hypothalamic lesions and intermittent explosive disorder. Journal of Neuropsychiatry \& Clinical Neurosciences, 4, 45-50. 
Treiber, F. A., Musante, L., Riley, W. T., \& Mabe, P. A. (1989). The relationship between hostility and blood pressure in children. Behavioral Medicine, 15, 173-178.

UrSin, H. (1960). The temporal lobe substrate of fear and anger. Acta Psychiatrica Scandinavica, 35, 378-396.

Weidner, G., Friend, R., Ficarrotto, T. J., \& Mendell, N. R. (1989). Hostility and cardiovascular reactivity to stress in women and men. Psychosomatic Medicine, 51, 36-45.

WeINSTEIN, S. (1978). Functional cerebral hemispheric asymmetry. In M. Kinsbourne (Ed.), Asymmetrical function of the brain. New York: Cambridge University Press.

WINER, B. J. (1971). Statistical principles in experimental design (2nd ed.). New York: McGraw-Hill.

WitTling, W. (1990). Psychophysiological correlates of human brain asymmetry: Blood pressure changes during lateralized presentation of an emotionally laden film. Neuropsychologia, 28, 457-470.

Woods, J. W. (1956). Taming of the wild in Norway rat by rhinocephalic lesions. Nature, $170,869$.

Yokoyama, K., Jennings, R., Ackles, P., Hood, P., \& Boller, F. (1987). Lack of heart rate changes during an attention demanding task after right hemisphere lesions. Neurology, 37, 624-630.

Zamrini, E. Y., Meador, K. J., Loring, D. W., Nichols, F. T., LeE, G. P., Figueroa, R. E., \& Thompson, W. O. (1990). Unilateral cerebral inactivation produces differential left/right heart rate responses Neurology, 40, 1408-1411.

(Manuscript received September 16, 1998; revision accepted for publication April 6, 2000.) 